\title{
PROFISSIONAIS DA SAÚDE PORTADORES DE Staphylococcus aureus - PERSISTÊNCIA E RESISTÊNCIA EVOLUTIVA
}

\author{
HEALTHCARE WORKERS CARRIERS OF Staphylococcus aureus - \\ PERSISTENCE AND EVOLUTIONARY RESISTANCE
}

\author{
Cesar Roberto Busato ${ }^{1}$, Juarez Gabardo ${ }^{2}$, Maria Terezinha Carneiro Leão ${ }^{3}$ \\ 1 Autor para contato: Santa Casa de Misericórdia e Universidade Estadual de Ponta Grossa \\ - UEPG, Departamento de Biologia Geral, Campus em Uvaranas, Ponta Grossa, PR, Brasil; \\ (42) 3220-3126; e-mail: crbusato@brturbo.com.br \\ 2 Universidade Federal do Paraná - UFPR, Departamento de Genética, Curitiba, PR \\ 3 Universidade Federal do Paraná - UFPR, Hospital das Clínicas, Curitiba, PR
}

Recebido para publicação em 25/09/2006

Aceito para publicação em 14/08/2007

\section{RESUMO}

Esta pesquisa fez um estudo prospectivo com análise estatística, com a finalidade de verificar a prevalência de portadores de Staphylococcus aureus em profissionais da saúde da Santa Casa de Misericórdia de Ponta Grossa (SCMPG), bem como sua persistência e variação da resistência. Foram colhidas em dois momentos, com intervalo de quatro anos, culturas de "swab nasal" em 105 profissionais da saúde sadios, que não tivessem feito uso de antibióticos ou antisépticos nasais até três semanas antes da coleta. As bactérias classificadas como Staphylococcus aureus foram submetidas a antibiograma pela técnica de Kirby e Bauer. Trinta e seis (34,2\%) apresentaram culturas positivas para Staphylococcus aureus na amostra 1, e $34(32,3 \%)$ na amostra 2 . Tais percentuais foram testados por meio do teste Tukey (d.m.s. $=7,74)$ e não apresentaram diferenças significativas. Dezenove profissionais com culturas positivas em ambas as amostras, configurando um grupo de portadores persistentes, tiveram suas cepas submetidas à antibiograma e a fagotipagem. Os percentuais de resistência testados mostraram diferença significativa em relação à penicilina (d.m.s. a 5\%=18,21). Seis $(31,5 \%)$ dos portadores persistentes apresentaram a fagotipagem cepas provavelmente relacionadas. Os profissionais da saúde apresentaram colonização nasal por Staphylococcus aureus semelhante em ambas as amostras.

Palavras-chave: Staphylococcus aureus; portadores de Staphylococcus aureus; profissionais da saúde 


\begin{abstract}
A prospective study and a statistic analysis were performed in order to verify the prevalence of Staphylococcus aureus carriers among health-care workers of the Santa Casa de Misericórdia de Ponta Grossa (SCMPG). The study was carried out in two different stages, with a four-year interval. The persistence and the resistance variation were also verified. Two samples of "Swab nasal" cultures were collected from 105 healthy health-care workers of the SCMPG who had not made use of antibiotics or nasal anti-septics in a period of three weeks before the collection. The bacteria classified as Staphylococcus aureus were submitted to an antibiogram through the Kirby and Bauer technique. Thirty six $(34,2 \%)$ of the first sample and thirty-four $(32,3 \%)$ of the second presented Staphylococcus aureus-positive cultures. Those percentages were also tested by means of the Tukey test (d.m.s $=7,74)$ and presented no significant differences. Nineteen professionals with positive cultures in the two samples were considered a group of persistent carriers and had their strains submitted to an antibiogram and to phagotyping. The group showed a significant variable resistance rate to the antibiotic penicillin (d.m.s. in $5 \%=18,21)$. Six out of nineteen $(31,5 \%)$ of the persistent carriers had phagotyping related strains in the two the samples. SCMPG Health-care workers showed nasal colonizations by Staphylococcus aureus, which were similar in both samples.
\end{abstract}

Key words: Staphylococcus aureus, Staphylococcus aureus carriers, healthcare workers

\section{Introdução}

Staphylococcus aureus continua sendo importante causa de infecção hospitalar, especialmente no sítio cirúrgico (Abboud; Firmino, 2000; Aha, 1974; Panlilio et al., 1992; Proahsa, 2000). Encontrado em recém natos, com 72 horas de vida, a bactéria coloniza o indivíduo, estabelecendo-se o estado de portador, que pode ser contínuo ou intermitente (Ako-Nai; Torimiro; Lamikanra, 1991). Apesar de ser encontrado nas mãos, nas axilas, no períneo e em quase toda superfície cutânea, é na cavidade nasal que sua presença é mais freqüente, mais intensa e mais duradoura (Santos; Solé-Vernin, 1981).

A condição de portador é expressão do tropismo tissular, e depende da inalação e fixação da bactéria a receptores moleculares da célula epitelial. A manutenção deste estado depende da disputa estabelecida entre a flora microbiana e os fatores de defesa do hospedeiro, o que divide os portadores em colonizados freqüentemente, algumas vezes colonizados e jamais colonizados (Gordon, 1993). Profissionais da saúde portadores podem, contaminar as mãos, e transmitir o organismo a outros pacientes (Boyce, 1996; Fascia et al., 2003). Surtos de infecção hospitalar por Staphylococcus aureus têm sido relatados e habitualmente, associados a fatores como: uso exagerado de antibióticos, inadequada lavagem das mãos, falta de cuidados da enfermagem e profissionais da saúde portadores (Sheretz et al., 1996).

A bactéria tem demonstrado taxas crescentes de cepas resistentes a oxacilina e aos beta-lactâmicos em geral, configurando o tipo MRSA(Methicillin Resistant Staphylococcus aureus), responsável por infecções mais graves e de maior custo de tratamento (Boyce, 1991; Kampf et al., 2003) MRSA tem sido relatado como endêmico com taxas entre 40 e $70 \%$ em infecções estafilocócicas em todo o mundo (Boyce, 1991; Lucet 
et al., 2003). A Dinamarca reduziu de 34 para menos de $1 \%$ a presença de MRSA e sustentou estes níveis por mais de 10 anos, através do controle do uso de antibióticos (seleção), pesquisa e isolamento de portadores (Monnet, 1999).

O paciente em decorrência da própria doença, da manipulação sofrida, da pressão de seleção pelo uso de antibióticos e da aquisição de bactérias hospitalares torna-se o principal reservatório e vítima da Infecção Hospitalar. Profissionais da saúde colonizados tornam-se disseminadores de bactérias resistentes (Fernandes; Fernandes; Ribeiro Filho, 2000; Geubbels et al., 2000). O reservatório para a transmissão da resistência é principalmente o colonizado, pois o infectado representa apenas a ponta do "iceberg" (Farr, 2000). Assim reconhecem-se dois tipos de Staphylococcus aureus em ambiente hospitalar: o permanente e o transitório. O primeiro é encontrado nos portadores que estão em permanente contato como são os profissionais da saúde e o meio ambiente. O segundo é encontrado nos pacientes infectados e portadores que se encontram em contato temporário com o hospital. O relacionamento que se estabelece entre os profissionais e os pacientes no dia a dia da atividade hospitalar pode fazer que ocorra uma renovação tanto nas cepas que compõem o permanente quanto o transitório. O Profissional da saúde inserido nesta cadeia epidemiológica tem sido visto apenas como vetor, todavia há pouca valorização na importância do seu papel para conhecimento da evolução da resistência da microbiota hospitalar.

\section{Material e métodos}

\section{Amostras}

Foram colhidas, com um intervalo de quatro anos (1996 e 2000), duas amostras de culturas de $s w a b$ nasal, em 105 profissionais da saúde da Santa Casa de Misericórdia de Ponta Grossa, todos sadios e que não tivessem feito uso de anti-sépticos nasais ou antibióticos até três semanas antes da data da coleta.

Os profissionais escolhidos para a pesquisa foram aqueles que entravam em contato direto com os pacientes do hospital.
As amostras foram obtidas pela fricção de zaragatoas umedecidas com soro fisiológico estéril na cavidade nasal em ambas as narinas e semeadura imediata.

\section{Isolamento}

O material obtido foi semeado diretamente nos meios de Baird - Parker Egg Volk- Tellurite Medium e Manitol SaltAgar, ambos seletivos (Oxoid), pela técnica de esgotamento e a seguir incubados em aerobiose a $37^{0}$ centígrados por 24 horas.

\section{Identificação}

As colônias suspeitas foram confirmadas microscopicamente pela coloração de Gram. Estas colônias foram repicadas no meio Manitol Salt Agar para realização da pesquisa da coagulase livre em tubo e teste de sensibilidade aos antibióticos, pela técnica de Kirby e Bauer, recomendada pelo NCCLS (National Committee for Clinical Laboratory Standards - USA) usando discos para antibiograma.

\section{Seleção de culturas para fagotipagem}

Dos 105 profissionais pesquisados, dezenove tiveram culturas positivas nas duas amostras que selecionadas e estas, foram submetidas à fagotipagem na Universidade de São Paulo pela técnica do Laboratório Internacional de referência sediado em Colindale -Londres.

\section{Medidas de controle}

Durante a fase do estudo, a Comissão de Controle de Infecção Hospitalar (CCIH) manteve as seguintes medidas: liberação de antibióticos mediante preenchimento de formulário; isolamento preventivo na admissão para pacientes infectados, oriundos de outro hospital ou com re-internações até resultado de cultura; isolamento de pacientes infectados por bactérias multirresistentes; descolonização de MRSA com mupirocin nasal, duas vezes ao dia, por um período de cinco dias em profissionais da saúde portadores; treinamento em serviço, visando a manutenção de normas de controle de infecção hospitalar. 


\section{Tratamento estatístico dos dados}

As percentagens observadas foram transformadas para arco seno raiz de " $\mathrm{x}$ " $(\mathrm{x}=$ percentagem $) \mathrm{e}$ comparadas pelo teste de Tukey.

\section{Resultados}

Dos cento e cinco profissionais pesquisados nas duas amostras, $36(34,2 \%)$ apresentaram culturas positivas para Staphylococcus aureus na amostra 1, e $34(32,3 \%)$ na amostra 2 . Tais percentuais foram testados através do teste Tukey (d.m.s. a $5 \%=7,74) \mathrm{e}$ não apresentaram diferenças significativas.

Dezenove (18\%) dos 105 profissionais pesquisados nas duas amostras apresentaram culturas positivas em ambas as ocasiões. Tabela 1.

As 36 culturas da primeira amostra e as 34 da segunda foram submetidas a antibiograma como mostra a tab. 2 .
Tabela 1 - Resultado das culturas dos profissionais pesquisados nas duas amostras

\begin{tabular}{lcc}
\hline & $\mathrm{N}$ & $\%$ \\
\hline $\begin{array}{l}\text { NEGATIVO } 1^{\mathrm{a}} \text { AMOSTRA } \\
\text { NEGATIVO } 2^{\mathrm{a}} \text { AMOSTRA }\end{array}$ & 54 & 51,4 \\
POSITIVO 1 & & \\
NEGATIVO $2^{\mathrm{a}}$ AMOSTRA & & \\
NEGATIVO $1^{\mathrm{a}}$ AMOSTRA & 17 & 16,1 \\
POSITIVO 2 & & \\
POSITIVO $1^{\mathrm{a}}$ AMOSTRA & 15 & 14,2 \\
POSITIVO $2^{\mathrm{a}}$ AMOSTRA & 19 & 18,0 \\
\hline TOTAL & 105 & 100 \\
\hline
\end{tabular}

Tabela 2 - Freqüência da resistência de Staphylococcus aureus nos profissionais pesquisados em ambas as amostras.

\begin{tabular}{lcccc}
\hline ANTIBIÓTICO & \multicolumn{2}{c}{ AMOSTRA 1} & \multicolumn{2}{c}{ AMOSTRA 2 } \\
\hline AMICACINA & $\mathrm{N}=36$ & $\%$ & $\mathrm{~N}=34$ & 11,7 \\
AMPICILINA & 05 & 13,8 & 04 & 97,0 \\
CEFALOTINA & 32 & 88,8 & 33 & 17,6 \\
CEFOTAXIMA & 05 & 13,8 & 06 & 17,6 \\
CIPROFLOXACINA & 06 & 16,6 & 06 & 29,4 \\
CLINDAMICINA & 04 & 11,1 & 10 & 20,5 \\
CLORANFENICOL & 06 & 16,6 & 07 & 23,5 \\
COTRIMOXAZOL & 09 & 25,0 & 08 & 20,5 \\
ERITROMICINA & 06 & 16,6 & 07 & 61,7 \\
GENTAMICINA & 14 & 38,8 & 21 & 14,7 \\
OXACILINA & 11 & 30,5 & 05 & 17,6 \\
PENICILINA & 05 & 13,8 & 06 & 100,0 \\
RIFAMPICINA & 33 & 91,6 & 34 & 8,8 \\
TETRACICLINA & 02 & 5,5 & 03 & 41,1 \\
TOBRAMICINA & 13 & 36,1 & 14 & 20,5 \\
VANCOMICINA & 13 & 36,1 & 07 & 0,0 \\
\hline
\end{tabular}


Os percentuais de resistência testados não demonstraram significância, (d.m.s.=13,42 a 5\%).

As culturas dos 19 profissionais, positivas para
Staphylococcus aureus nas duas amostras foram submetidas a teste de sensibilidade aos antimicrobianos. Tabela 3.

Tabela 3 - Resistência de Staphylococcus aureus nos profissionais com culturas positivas em ambas as amostras.

\begin{tabular}{lcccc}
\hline ANTIBIÓTICO & & AMOSTRA 1 & AMOSTRA 2 \\
\hline AMICACINA & 02 & $\%$ & 03 & $\%$ \\
AMPICILINA & 18 & 10,5 & 19 & 15,7 \\
CEFALOTINA & 03 & 94,7 & 03 & 100,0 \\
CEFOTAXIMA & 03 & 15,7 & 03 & 15,7 \\
CIPROFLOXACINA & 02 & 15,7 & 05 & 15,7 \\
CLINDAMICINA & 02 & 10,5 & 04 & 26,3 \\
CLORANFENICOL & 06 & 10,5 & 04 & 21,0 \\
COTRIMOXAZOL & 03 & 31,5 & 03 & 15,7 \\
ERITROMICINA & 07 & 15,7 & 10 & 52,6 \\
GENTAMICINA & 05 & 36,8 & 03 & 15,7 \\
OXACILINA & 03 & 26,3 & 03 & 15,7 \\
PENICILINA & 17 & 15,7 & 19 & 100,0 \\
RIFAMPICINA & 01 & 89,4 & 01 & 5,2 \\
TETRACICLINA & 08 & 5,2 & 08 & 42,1 \\
TOBRAMICINA & 07 & 42,1 & 03 & 15,7 \\
VANCOMICINA & 00 & 36,8 & 00 & 0,0 \\
\hline
\end{tabular}

Os percentuais de resistência testados mostraram diferença significativa em relação à penicilina (d.m.s. a $5 \%=18,21)$.

Apenas um profissional da saúde, apresentou em ambas as ocasiões cultura com resistência à oxacilina, configurando um Staphylococcus aureus meticilino- resistente MRSA.

As culturas dos 19 profissionais, positivas para Staphylococcus aureus nas duas amostras, foram submetidas a fagotipagem pelo Laboratório de Ciências Farmacêuticas da Universidade de São Paulo, à procura de uma identidade entre si. Tabela 4. 
Tabela 4 - Comparação através de fagotipagem de cepas de Staphylococcus aureus encontrada nos profissionais da saúde em ambas as amostras

\begin{tabular}{llll}
\hline NÚMERO & FAGÓTIPO A1 & FAGÓTIPO A 2 & RELAÇÃO \\
\hline 01 & I, III, FE & III, FE & ALTAMENTE RELAC. \\
15 & I, III, FE, NC & I & NÃO RELACIONADO. \\
29 & II & I & NÃO RELACIONADO. \\
38 & I, III, FE & NHL & NÃO RELACIONADO. \\
53 & III & I & NÃO RELACIONADO \\
90 & III, FE & I & NÃO RELACIONADO. \\
91 & III & NHL & NÃO RELACIONADO \\
118 & I, III, FE & I, FE & NÃO RELACIONADO. \\
121 & NHL & I & NÃO RELACIONADO. \\
135 & II & II & ALTAMENTE RELAC. \\
173 & FE & FE & NÃO RELACIONADO \\
179 & NHL & NHL & PROVAVELM. RELAC. \\
184 & NC, FE & I & NÃO RELACIONADO. \\
189 & NHL & I & NÃO RELACIONADO \\
193 & I & I & ALTAMENTE RELAC. \\
215 & NHL & I & NÃO RELACIONADO \\
232 & NHL & NHL & PROVAVELM. RELAC. \\
260 & II & III & NÃO RELACIONADO \\
NAP & III , V & ALTAMENTE RELAC. \\
\hline
\end{tabular}

Seis profissionais da saúde apresentaram à fagotipagem, em ambas as amostras, padrões de lise alta e provavelmente relacionados configurando portadores persistentes da mesma bactéria ou dela originada; estes, submetidos ao antibiograma mostraram a variação de resistência encontrada na tabela 5. 
Tabela 5 - Resistência de Staphylococcus aureus de mesmo fagótipo dos profissionais de saúde com culturas positivas em ambas as amostras

\begin{tabular}{lcrcr}
\hline ANTIBIÓTICO & \multicolumn{2}{c}{ AMOSTRA 1} & \multicolumn{2}{c}{ AMOSTRA 2 } \\
\hline AMICACINA & $\mathrm{N}=6$ & $\%$ & 00 & 0,0 \\
AMPICILINA & 00 & 100,0 & 06 & 100,0 \\
CEFALOTINA & 06 & 0,0 & 00 & 0,0 \\
CEFOTAXIMA & 00 & 0,0 & 00 & 0,0 \\
CIPROFLOXACINA & 00 & 0,0 & 01 & 16,6 \\
CLINDAMICINA & 01 & 16,6 & 01 & 16,6 \\
CLORANFENICOL & 02 & 33,3 & 01 & 16,6 \\
COTRIMOXAZOL & 01 & 16,6 & 00 & 0,0 \\
ERITROMICINA & 04 & 66,6 & 03 & 50,0 \\
GENTAMICINA & 01 & 16,6 & 00 & 0,0 \\
OXACILINA & 00 & 0,0 & 00 & 0,0 \\
PENICILINA & 06 & 100,0 & 06 & 100,0 \\
RIFAMPICINA & 00 & 0,0 & 00 & 0,0 \\
TETRACICLINA & 03 & 50,0 & 04 & 66,6 \\
TOBRAMICINA & 02 & 33,3 & 00 & 0,0 \\
VANCOMICINA & 00 & 0,0 & 00 & 0,0 \\
\hline
\end{tabular}

\section{Discussão}

Dezenove profissionais de saúde portadores de Staphylococcus aureus nas amostras $1 \mathrm{e} 2$, configuram um grupo de portadores persistentes da bactéria de $18,0 \%$, em relação aos 105 pesquisados nas duas amostras.

Profissionais da saúde, portadores nasais, representam uma importante reserva de Staphylococcus aureus; aproximadamente $25 \%$ são portadores estáveis, 30 a $50 \%$ deles possuem a bactéria também nas mãos (Boyce, 1991).

O uso extensivo de antibióticos no hospital, faz com que a bactéria manifeste sua resistência também, no meio ambiente. Assim, pacientes e profissionais da saúde, estão expostos indiretamente à ação dos antimicrobianos, que atuam na microbiota endógena sele- cionando bactérias resistentes que se espalham através do contato individual (Mc Gowan, 1983).

A nítida associação entre a introdução de um antibiótico e os determinantes que conferem resistência, freqüentemente, é interpretada como causa, quando na realidade sugere seleção. A habilidade de um antibiótico em selecionar cepas resistentes depende de sua atividade letal intrínseca contra as espécies sensíveis, da habilidade do microrganismo de mudar o receptor ou utilizar genes de defesa, da proximidade de outras espécies capazes de transferir determinantes de resistência, e da habilidade de tolerar e expressar a resistência adquirida (John;Rice, 2000).

McGovan, em 1983 refere que o aumento da colonização ou infecção por microrganismos resistentes ocorre, quando no ambiente hospitalar, houve grande consumo de antibióticos, mesmo em indivíduos que não 
receberam a droga; modelos biológicos têm mostrado seleção bacteriana pela presença ambiental do antibiótico que produz marcados efeitos sobre a microbiota endógena e ambiental.

Embora não se possa quantificar o limiar de resistência de Staphylococcus aureus aos antibióticos testados para o ambiente da SCMPG, considera-se que a variação da resistência desta população $(n=19)$ comum às duas amostras representa no período de quatro anos, a pressão de seleção do ambiente hospitalar, para a bactéria.

Notam os dados supracitados, certa estabilidade na resistência aos antibióticos mais utilizados no tratamento à bactéria, como são oxacilina, cefalotina, cefotaxima, cotrimoxazol, vancomicina, rifampicina e, ainda, cloranfenicol e amicacina, bem como uma diminuição da resistência à gentamicina e tobramicina. Verificouse uma tendência de aumento na resistência à ciprofloxacina e a clindamicina. Os níveis de resistência à ampicilina, eritromicina, penicilina e tetraciclina fizeram com que estas drogas deixassem de ser utilizadas na sua terapêutica em nosso meio.

A fagotipagem mostrou que 6 dos 19 profissionais mantinham cepas provavelmente relacionadas durante o período do estudo e 13 eram portadores de cepas não-relacionadas às da primeira pesquisa..

Bactérias de fagótipos diferentes pressupõe uma substituição de cepas, cuja variação de resistência mantém-se próxima das tendências não-significativas da sub-amostra anterior $(\mathrm{n}=19)$, o que configura certa estabilidade na resistência bacteriana das cepas disponíveis de Staphylococcus aureus no ambiente da SCMPG.

Staphylococcus aureus de fagótipos relacionados são originados do mesmo ancestral e na subamostra $(n=6)$ configuram portadores habituais da mesma bactéria. Eventuais alterações no padrão de resistência destes microrganismos podem estar relacionadas a perda ou ganho de material genético como plasmídeos; transposons; integrons, através de transformação; transdução, pela ação de bacteriófagos e conjugação ou mutação (Brooks, Butel; Morse, 1998). Dois dos seis portadores apresentaram idêntico padrão de resistência nos antibiogramas e quatro mostraramse próximos das tendências não-significativas da subamostra dos portadores persistentes $(n=19)$. O material genético disponível para transposições é semelhante ao encontrado nas cepas disponíveis na SCMPG, com exceção das meticilino-resistentes, visto que neste subgrupo $(\mathrm{n}=6)$ não verificamos resistência à oxacilina. $\mathrm{O}$ que pode ser explicado pela descolonização realizada no período, nos portadores de MRSA.

Apenas 1 profissional comum às duas amostras estava colonizado por MRSA, porém de fagótipo diferente.

Embora a fagotipagem esteja sendo substituída pela eletroforese de DNA em campo pulsado trata-se de método que pode ser utilizado quando se pretende comparar amostras recentes com outras já fagotipadas, como é o caso do presente estudo.

\section{Conclusão}

Profissionais da saúde da SCMPG portadores persistentes de Staphylococcus aureus demonstraram em um intervalo de quatro anos a presença da cepa original em $1 / 3$ dos casos $(31,5 \%)$ e uma renovação em $2 / 3(68,5 \%)$, demonstrados pela fagotipagem. A variação da resistência à maioria dos antibióticos não foi significativa e traduzuma estabilidade na sensibilidade das cepas disponíveis no ambiente da Santa Casa de Misericórdia de Ponta Grossa.

\section{REFERÊNCIAS}

1. ABBOUD, C.S.; FIRMINO, A.L. Evaluation of hospital infection rates and control measures in a cardiac surgery hospital: 10 years experience. Infect Control Hosp Epidemiol. v.21, p.494-495, 2000.

2. AKO-NAI,A.K.; TORIMIRO, S.E.; LAMIKANRA,A., et al. A survey of nasal carriage of Staphylococcus aureus in a neonatal ward in Ile-Ife, Nigeria. Annals of Tropical Paediatrics, v.11, n.1,p.41-45, 1991.

3. AMERICAN HOSPITALASSOCIATION (AHA). Infection control in the hospital. Chicago: AHA, p.203, 1974.

4. BOYCE, J.M. Should we vigorously try to contain and control methicillin-resistant Staphylococcus aureus? Infect Control Hosp Epidemiol., v.12, p.46-54, 1991.

5. BOYCE, J.M. Preventing Staphylococcal infections by eradicating nasal carriage of Staphylococcus aureus: Proceeding with caution. Infect Control Hosp Epidemiol., v.17, p.775-779, 1996. 
6. BROOKS, G.F; BUTEL, J.S; MORSE, S.A. Medical Microbiology. Stanford, Connecticut: Appleton \& Lange, 1998, p. 93-97.

7. FARR, B.M. Reasons for noncompliance with infection control guidelines. Infect Control Hosp Epidemiol., v.21, p.411$416,2000$.

8. FASCIA, P.; MARTIN, I; MALLAVAL, F.O.; GRATTARD, F.; POZZETTO,B; LUCHT,F; BHERTELOT, P. Possible implication of student nurses in the transmission of MRSA during a nosocomial outbreak. Pathol Biol; 51(8-9):479-82,2003

9. FERNANDES,A.T.; FERNANDES, M.O.V.; RIBEIROFILHO, N. Infecção Hospitalar e suas Interfaces na área da Saúde. São Paulo: Editora Atheneu, 2000, p.104-105.

10. GEUBBELS, E.L.P.E.; GROOTA, J.M.; BERG, J.M.J.V.D.; BOER, A.S. An operating surveillance system of surgical-site infections in the Netherlands: results of the PREZIES national surveillance network. Infect Control Hosp Epidemiol., v.21, p.311-318, 2000 .

11. GORDON, J. Clinical significance of methicillin-sensitive and methicillin-resistant Staphylococcus aureus in UK hospitals and the relevance of povidone-iodine in their control. Postgrad. Med. J., v.69, supp. 1.3, p.s106-s116,1993.

12. JOHN, JR. J.F.; RICE, L.B. The microbial genetics of antibiotic cycling. Infect Control Hosp Epidemiol., v.21 (Suppl.):s22-s31, 2000.

13. KAMPF,G; ADENA,S; RÜDEN, H; WEIST,K. Inducibility and potential role of MecA-gene-positive oxacillin-susceptible Staphylococcus aureus from colonized healthcare workers as a source for nosocomial infections. J Hosp Infect; 54(2):124-
9.2003.

14. LUCET,JC;CHEVRET,S; DURAND-ZALESKI,I; CHASTANG,C; REGNIER,B. Prevalence and risk factors for carriage of MRSA at admission to the intensive care unit: results of a multicenter study. Arch Intern Med; 163 (2):181-8, 2003.

15. McGOWAN, Jr. J.E. Antimicrobial resistance in hospital organisms and its relation to antibiotic use. Rev Infect Dis., v.5,p.1033-1048, 1983.

16. MONNET, D.L. Plenary Session. Global conference on antibiotic resistance; march 1999; Toronto, Ontario, Canada.

17. PANLILIO, A.; CULVER, D.H.; GAYNES, R.P.; BANERJEE, S.;HENDERSON, T.S.; TOLSON, J.S.; MARTONE, W.J. Methicillin-resistant Staphylococcus aureus in U.S. hospitals, 1975-1991. Infect Control Hosp Epidemiol., v.13, p.582-586, 1992.

18. PROAHSA - Boletim de indicadores. Programa de Estudos Avançados em Administração Hospitalar e de Sistemas de Saúde do HC da FMUSP e da EAESP da Fundação Getúlio Vargas, v.8, p.1-4, 2000.

19. SANTOS, B.M.O.; SOLÉ-VERNIN, C. Papel epidemiológico dos portadores sãos de Staphylococcus aureus como fonte de infecção. Rev. Inst. Med. Trop., São Paulo, v.23, n.5, p.217224, 1981.

20. SHERETZ, R.J.; REAGAN, D.R.; HAMPTON, K.D.; ROBERTESON, K.L.; STREED, A.S.; HOEN, H.M.; THOMAS, R.; GWALTNEY, JR. J.M. A cloud adult: The Staphylococcus aureus-virus interaction revisited. Ann Intern Med; v.(124), p.539-547, 1996. 